\title{
CARDINAL AND ORDINAL INCONSISTENCY IN THE PAIRWISE COMPARISONS MATRIX
}

\author{
KONRAD KULAKOWSKI \\ AGH UNIVERSITY OF SCIENCE AND TECHNOLOGY, KRAKÓW, POLAND \\ kkulak@agh.edu.pl
}

Keywords: AHP, Kendall Babington Smith index, inconsistency

\begin{abstract}
The Analytic Hierarchy Process proposed by Saaty in his seminal work (Saaty 1977) is a method for representing any decision problem in the form of a hierarchy. It divides a problem into sub-problems and then leads experts through a series of paired judgments to present them at the end the numerical ranking of all considered alternatives. Of course, not all judgments may be consistent. It means that for the given PC matrix $A=\left[a_{i j}\right]$ there are exists at least three entries $a_{i k}, a_{k j}$ and $a_{i j}$ such that $a_{i k} a_{k j} \neq a_{i j}$. Inconsistency is the greater, the more there are such triads and also the more significant the difference between $a_{i k}, a_{k j}$ and $a_{i j}$. It is proven that $C I$ - the consistency index defined by Saaty in (Saaty 1977)

$$
C I=\frac{\lambda_{\max }-n}{n-1}
$$

is a good indicator of inconsistency for PC matrices of size $n \times n\left(\lambda_{\max }\right.$ is the principal eigenvalue of $A$ ). In order to eliminate the effect of the size of the matrix and objectify the results, Saaty introduced CR - consistency ratio given as

$$
C R=\frac{C I}{R_{n}},
$$

where $R_{n}$ - random index, defines the average value of $C I$ for the randomly chosen PC matrices of size $n \times n$. CI measures inconsistency quantitatively. This means that it takes into account not only the fact that $a_{i k} a_{k j} \neq a_{i j}$ but also it takes into account how far $a_{i k} a_{k j}$ is from $a_{i j}$. Inconsistency can also be understood qualitatively. In this approach, it is important whether the preference relation between three alternatives is transitive. For example, if an alternative $X_{i}$ is more preferred than the alternative $X_{k}$ (denoted as $X_{i} \succ X_{k}$ ) and an alternative $X_{k}$ is more preferred than the alternative $X_{j}$ (denoted as $X_{k} \succ X_{j}$ ) then we may expect that also $X_{i} \succ X_{j}$. The opposite situation will be seen as inconsistency. Similarly, if there is a tie between $X_{i}$ and $X_{k}$, i.e., $X_{i} \sim X_{k}$ and $X_{k} \succ X_{j}$ International Symposium on the


but at the same time $X_{i} \prec X_{j}$ or $X_{i} \sim X_{j}$ we also feel the comfort of inconsistency. This way of thinking about inconsistency is called the qualitative approach. Qualitative (ordinal) inconsistency can also be measured. One of the indexes used for this purpose is Kendall's inconsistency index $\zeta$ :

$$
\zeta(A)=1-\frac{|A|}{\mathcal{I}(n)}
$$

where $|A|$ is the number of intransitive triads described by the matrix $A$ and $\mathcal{I}(n)$ :

$$
\mathcal{I}(n)=\left\{\begin{array}{ll}
\frac{n^{3}-n}{24} & \text { when } n \text { is odd } \\
\frac{n^{3}-4 n}{24} & \text { when } n \text { is even }
\end{array},\right.
$$

represents the maximal possible number of intransitive triads in the ordinal PC matrix of size $n \times n$ (Kendall 1940). Unfortunately, Kendall's formula does not cover paired comparisons with ties. It turns out that when ties are allowed the maximal possible number of intransitive triads increases, and then the formula $\mathcal{I}(n)$ need to be replaced by other compatible with pairwise comparisons with ties. The desired replacement for $\mathcal{I}(n)$ is:

$$
\mathcal{Y}(n)=\left(\begin{array}{l}
n \\
3
\end{array}\right)-\left(\left(\left\lfloor\begin{array}{c}
n / 2\rfloor \\
3
\end{array}\right)-\mathcal{I}(\lfloor n / 2\rfloor)\right)-\left(\left(\left[\begin{array}{c}
n / 2\rceil \\
3
\end{array}\right)-\mathcal{I}(\lceil n / 2\rceil)\right) .\right.\right.
$$

The above formula has been proposed by the author in (Kułakowski 2018). Therefore the generalized Kendall's inconsistency index takes the form:

$$
\zeta_{g}(A)=1-\frac{|A|}{\mathcal{Y}(n)} \text {. }
$$

What is important $\zeta_{g}$ can also be used with quantitative paired comparisons; hence, it allows assessing ordinal inconsistency of PC matrices used in AHP. For this purpose, it is enough to accept that wherever for some $A=\left[a_{i j}\right]$ holds that $a_{i j}>1$ then $X_{i} \succ X_{j}$, $a_{i j}<1$ then $X_{i} \prec X_{j}$, and $a_{i j}=1$ then $X_{i} \sim X_{j}$.

It is easy to prove that if $A$ is consistent quantitatively then also it is consistent qualitatively, i.e. $\operatorname{CI}(A)=0$ implies that $\zeta_{g}(A)=1$. However, the question arises what happens when A gets more and more inconsistent. To answer this question a series of Monte-Carlo experiments were carried out. The experiment consisted of 781 steps. In every step 100 randomly disturbed matrices were drawn, where the level of disturbance increased with each step. The average value of $C I$ and $\zeta_{g}$ was calculated at each step. Of course, the increase of quantitative inconsistency represented by $C I$ was accompanied by an increase in qualitative inconsistency $\zeta_{g}$. However, the increase in inconsistency for both indices looked different. While $C I$ raised regularly and almost linearly at the same time $\zeta_{g}$ 's decline stopped near the value 0.85 (Fig. 1). 
ISAHP Article: A Style Guide for Paper Proposals To Be Submitted to the International Symposium on the Analytic Hierarchy Process 2018, Hong Kong, HK.

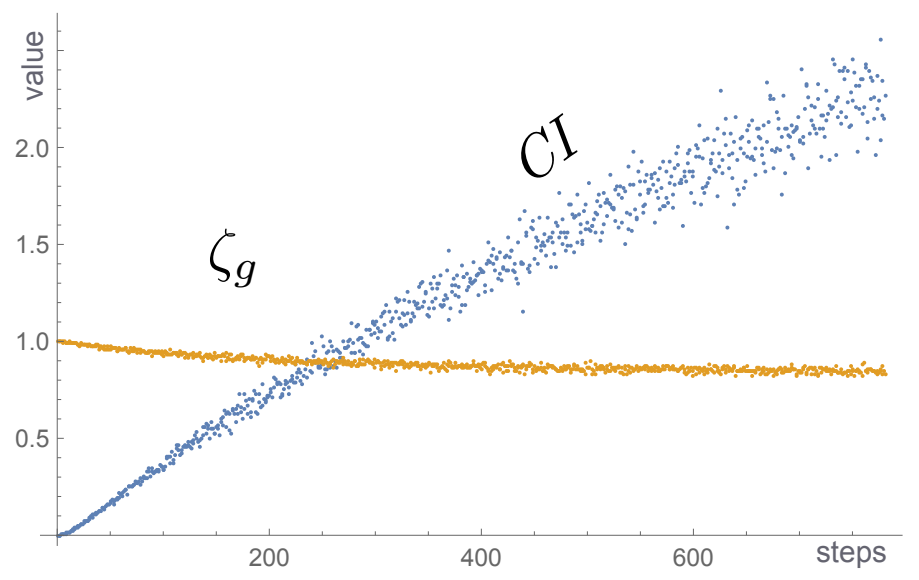

Fig. 1. The average values of $C I$ and $\zeta_{g}$ for the increasing level of disturbance.

The above figure shows different dynamics of both indexes. Hence, both indexes need to be treated and interpreted differently. In particular, it is easy to observe that even a large quantitative inconsistency does not translate into a considerable qualitatively inconsistency. This somewhat surprising observation raises the question of the meaning of qualitative inconsistency for AHP. The paper is an attempt to look for answers.

Saaty, T.L. (1977). A scaling method for priorities in hierarchical structures. Journal of Mathematical Psychology, 15, 234-281.

Kendall M. G., \& Babington Smith B. (1940). On the Method of Paired Comparisons. Biometrika, Vol. 31, No. 3/4, pp. 324-345

Kułakowski K. (2018). Inconsistency in the ordinal pairwise comparisons method with and without ties. European Journal Of Operational Research, doi.org/10.1016/j.ejor.2018.03.024 